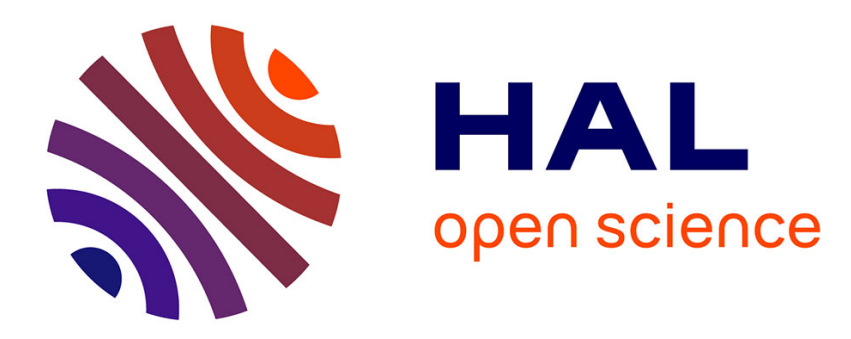

\title{
Ce que l'histoire de la psychiatrie nous dit de la psychanalyse
}

Benoît Eyraud, Livia Velpry

\section{To cite this version:}

Benoît Eyraud, Livia Velpry. Ce que l'histoire de la psychiatrie nous dit de la psychanalyse. Revue du MAUSS, 2011, 37, pp.103-120. 10.3917/rdm.037.0103 halshs-00910942

\section{HAL Id: halshs-00910942 \\ https://shs.hal.science/halshs-00910942}

Submitted on 10 Mar 2014

HAL is a multi-disciplinary open access archive for the deposit and dissemination of scientific research documents, whether they are published or not. The documents may come from teaching and research institutions in France or abroad, or from public or private research centers.
L'archive ouverte pluridisciplinaire HAL, est destinée au dépôt et à la diffusion de documents scientifiques de niveau recherche, publiés ou non, émanant des établissements d'enseignement et de recherche français ou étrangers, des laboratoires publics ou privés. 


\title{
CE QUE L'HISTOIRE DE LA PSYCHIATRIE NOUS DIT DE LA PSYCHANALYSE
}

\author{
Benoît Eyraud et Livia Velpry
}

\section{La Découverte | Revue du MAUSS}

\author{
2011/1 - n 37 \\ pages 103 à 120
}

\section{ISSN 1247-4819}

Article disponible en ligne à l'adresse:

http://www.cairn.info/revue-du-mauss-2011-1-page-103.htm

Pour citer cet article :

Eyraud Benoît et Velpry Livia, "Ce que l'histoire de la psychiatrie nous dit de la psychanalyse », Revue du MAUSS, 2011/1 n³7, p. 103-120. DOI : 10.3917/rdm.037.0103

Distribution électronique Cairn.info pour La Découverte.

(c) La Découverte. Tous droits réservés pour tous pays.

La reproduction ou représentation de cet article, notamment par photocopie, n'est autorisée que dans les limites des conditions générales d'utilisation du site ou, le cas échéant, des conditions générales de la licence souscrite par votre établissement. Toute autre reproduction ou représentation, en tout ou partie, sous quelque forme et de quelque manière que ce soit, est interdite sauf accord préalable et écrit de l'éditeur, en dehors des cas prévus par la législation en vigueur en France. II est précisé que son stockage dans une base de données est également interdit. 


\title{
Ce que l'histoire de la psychiatrie nous dit de la psychanalyse
}

\author{
Benoît Eyraud et Livia Velpry
}

Pendant de nombreuses années, la psychanalyse a exercé une grande influence sur l'ensemble des sciences humaines et plus spécifiquement sur toutes les questions relatives au monde psy. Plus récemment, cette influence s'est érodée au profit d'autres domaines de connaissance sur le psychisme : neurosciences, psychologie cognitive et comportementale, au motif principalement d'une prétendue plus grande efficacité thérapeutique que ces domaines du savoir permettraient d'atteindre. Le rapport Psychothérapie : trois approches évaluées [Inserm, 2004] constitue un exemple emblématique de ce point de vue. Aujourd'hui, les critiques de la psychanalyse portent donc d'abord sur son efficacité thérapeutique. Derrière cette critique pratique, il nous semble cependant qu'il existe une mise en cause de la vision psychanalytique du monde, qui réside notamment dans l'articulation problématique de certains idéaux démocratiques, comme celui de l'égalité, avec la vision anthropologique portée par la psychanalyse. Dans l'optique de participer aux réflexions développant une vision alternative à celle portée par les théoriciens utilitaristes, nous proposons de mieux identifier les raisons des difficultés rencontrées aujourd'hui par le discours psychanalytique à partir d'une mise en perspective historique des discours structurant l'organisation du soin dans les établissements psychiatriques. 
Notre contribution fait le pari que l'institution psychiatrique est un excellent révélateur des enjeux qui sont aujourd'hui posés à la psychanalyse, aux politiques de santé, et plus généralement aux sciences humaines, dans leur volonté de développer une anthropologie « anti-utilitariste ». Depuis son émergence, l'institution psychiatrique se caractérise en effet par le fait qu'elle se doit de porter une vision anthropologique afin de justifier de ses pratiques thérapeutiques, dont les résultats manquent d'évidence pratique [Gauchet, 1980]. Il nous semble que, pendant un temps, la référence à la psychanalyse permettait précisément d'articuler vision anthropologique et pratique thérapeutique. Mais cette référence s'est affaiblie devant l'émergence de nouvelles configurations, dont l'idéologie contemporaine de l'évaluation constitue sans doute la forme la plus forte. Progressivement, les logiques gestionnaires se sont imposées.

Pour bien saisir comment ces logiques ont réussi à faire concurrence à la vision portée par les psychanalystes, et dans bien des domaines, à s'imposer, il s'agira de montrer qu'elles sont adossées à une vision utilitariste de la santé, et au souci de la démocratie sanitaire. Loin d'être anecdotiques, il nous semble que les soubassements idéologiques de cette vision gestionnaire de la santé doivent être explicités afin de mieux saisir les manières dont le discours psychanalytique pourrait porter aujourd'hui une vision moins utilitariste de la démocratie, attentive à reconnaître les singularités de la personne humaine ${ }^{1}$.

1. Le texte vise à ouvrir une perspective de recherche originale sur un domaine qui reste encore largement à explorer, les travaux sociohistoriques sur l'histoire de la psychanalyse en France étant encore peu importants. Il ne vise pas à rendre compte de la multitude des débats et controverses internes à la théorie psychanalytique ou produits dans le cadre des recompositions actuelles des politiques de santé. Il veut stimuler une réflexion plutôt qu'exposer une démonstration et s'appuie pour cela sur l'analyse de matériaux hétérogènes - archives d'établissement, ouvrages de référence pour les praticiens, travaux d'historiens et de sociologues - qui ont été retenus en raison de leur portée heuristique. 


\section{Le succès de l'extraterritorialité de la psychanalyse dans les institutions psychiatriques}

La diffusion de la psychanalyse a été longue à se mettre en place en France, ce qui explique son succès tardif auprès des psychiatres des hôpitaux. Certes, la première consultation psychanalytique est créée dans les années 1920 par René Laforgue dans le service universitaire de clinique des maladies mentales à l'hôpital Ste Anne, mais c'est une certaine hostilité des médecins qui domine alors [Widlöcher, 2004]. Cette expérience pionnière ne rencontre pas véritablement d'écho alors que le développement de la psychanalyse dans des institutions psychiatriques prend de l'ampleur dans plusieurs pays ${ }^{2}$. Il faut attendre le milieu des années 1950 pour que de nouvelles expériences soient développées, comme à la clinique de La Borde, là encore d'abord en marge des services régis par le cadre asilaire, ou par des intervenants se présentant comme psychologues [Ohayon, 2008]. La psychanalyse s'inscrit dans la suite d'un mouvement de réforme de l'hôpital psychiatrique qui se développe après l'introduction des sociothérapies, plus connues en France sous le terme de «psychothérapie institutionnelle», et des thérapies médicamenteuses.

\section{L'introduction difficile de la cure psychanalytique}

Cette intégration de la psychanalyse se présente d'abord comme l'introduction d'une nouvelle thérapeutique à l'intérieur de l'hôpital, améliorant le soin des malades. Elle y est associée à l'introduction des médicaments psychotropes : «En agissant sur les symptômes les plus graves, les neuroleptiques rendent accessibles à une approche psychothérapique des psychoses des malades auparavant coupés de ce genre d'approche » [Widlöcher, 2004, p. 12]. C'est en tout cas l'explication de «bon sens » [Lebovici, 1970, p. 47] apportée dans un premier temps pour justifier la présence de psychanalystes à l'intérieur de l'hôpital. C'est ainsi que Jean Bergeret défend son

2. La réception de la psychanalyse dans le monde psychiatrique s'est d'abord réalisée par l'entremise de Bleuler et des psychiatres de la Clinique de Burghözli à Zurich. Pour les États-Unis, voir par exemple Shorter E., A History of Psychiatry : from the Era of the Asylum to the Age of Prozac, John Wiley \& Sons, New York City, 1997. 
projet en prenant ses fonctions de médecin-psychologue à l'hôpital du Vinatier en 1957. Il développe sa vision propre de la psychothérapie auprès des médecins chefs de service en proposant une cure au cours de laquelle « tout ce que le malade a vécu de malheureux autrefois, sans s'en rendre compte, et qui l'a conduit là où il est, tout cela il faut essayer de le revivre ensemble pour l'en débarrasser » [Bergeret, 1964]. Cette cure suppose la répétition régulière des séances, si possible plusieurs fois par semaine. Mais ce bon sens se confronte rapidement aux contraintes de l'hôpital psychiatrique. Dès 1961, Bergeret explique par exemple qu'il abandonne progressivement les psychothérapies individuelles, «trop luxueuses » en termes de temps, au profit de psychothérapies de groupe réunissant deux fois par semaine pour une durée d'une heure une dizaine de malades de services différents.

Ces difficultés ne sont pas propres à l'hôpital du Vinatier ; tous les promoteurs de la psychanalyse dans les institutions psychiatriques les rencontrent. Même dans l'expérience menée dans le $\mathrm{XIII}^{\mathrm{e}}$ arrondissement de Paris, qui tirait une notoriété particulière du travail psychanalytique et le plaçait au centre de ces outils thérapeutiques, les difficultés à intégrer la cure dans le fonctionnement des structures étaient telles que les psychothérapeutes orientèrent progressivement leur activité vers « un rôle de réflexion et de théorisation sur le fonctionnement général de l'association » [Henckès, 2005, p. 78-85]. Ces obstacles font l'objet de nombreux débats dans les revues psychiatriques, comme l'Évolution psychiatrique ou l'Information psychiatrique, à la fin des années 1960 et au début des années 1970. Elles sont résumées de manière synthétique dans l'ouvrage collectif publié sous la direction de Racamier, Le Psychanalyste sans divan [1970], qui se présente comme un manifeste pour la diffusion de la psychanalyse dans les institutions psychiatriques.

La première difficulté réside dans le fait que l'institution psychiatrique héberge de nombreux patients psychotiques pour lesquels la «psychanalyse n'est guère indiquée, sinon à titre de méthode expérimentale ». L'efficacité de la théorie psychanalytique pour les patients psychotiques constitue un problème majeur débattu au sein de la communauté psychanalytique depuis les premières discussions de Freud avec Binswanger. Elle constitue de fait un défi pour les psychiatres hospitaliers et une littérature assez importante 
se développe autour de cette difficulté à partir notamment de l'expérience de Chesnut Lodge, où se pratique la psychanalyse avec des patients psychotiques dès les années 1940 [Bullard, 1939] ${ }^{3}$. La seconde est économique, et réside dans le fait que la cure psychanalytique est un luxe qui ne pourrait être mise en œuvre que pour un nombre limité de cas, "dérisoire », par rapport à la pression des besoins. Or, son usage ne peut être justifié par le traitement des cas de «névrose », qui précisément ne sont pas les plus difficiles à traiter pour l'institution psychiatrique. La troisième est organisationnelle et réside dans le statut de faveur qui serait de fait accordé aux psychanalystes travaillant, dans le cadre de la cure, dans des « conditions infiniment plus favorables que le reste de l'équipe psychiatrique » [Racamier, p. 45-65].

\section{La vision du monde des psychiatres-psychanalystes réformateurs}

Les promoteurs de la psychanalyse ont ainsi bien conscience que sa diffusion dans les institutions psychiatriques ne peut pas reposer sur les résultats de cures analytiques. Pour répondre à cette difficulté, une justification d'une autre nature doit être apportée. Plusieurs arguments sont présentés par les auteurs. Le premier est «scientifique ». La théorie psychanalytique est une « source de compréhension des maladies mentales » et elle permet d'accéder à une certaine « vérité de la folie » [Mannoni, 1970]. Diaktine rappelle ainsi que Freud a utilisé « à chaque étape de son œuvre des données provenant de l'étude des psychoses », et a établi que « le délire est le seul mode possible d'organisation qui reste possible pour le malade, compte tenu de son état antérieur, et des modifications qui y ont mis fin » [Diaktine, p. 26-27]. Le second argument est institutionnel. La psychanalyse constitue un outil de vigilance vis-à-vis des tendances « défensives » des organisations et des institutions. Elle s'inscrit ce faisant de manière revendiquée à la suite des promoteurs de la psychothérapie institutionnelle [Racamier, 1970]. La référence psychanalytique doit même, plus précisément, permettre de dépasser les limites d'une psychothérapie institutionnelle qui s'est « laissé mystifier par les instruments que

3. On peut mentionner également les travaux de Fromm-Reichmann [1948]. 
la société met à la disposition du psychiatre » [Diaktine, 1970, p. 28]. Incorporée à cette pratique, la psychanalyse sera d'ailleurs prise comme modèle par certains psychiatres du mouvement de la psychothérapie institutionnelle [Ehrenberg, 2010]. Mais la clef de voûte de l'argumentation réside dans la reconnaissance de la singularité de chaque malade : «Pour le psychanalyste au contraire, chaque malade fait l'objet d'une expérience unique, incomparable » [Lebovici, 1970, p. 49]. Une formule répandue alors synthétise la place prise par le psychanalyste dans l'institution psychiatrique : « ce qu'il est compte plus que ce qu'il dit ».

\section{Les conditions sociales de l'extraterritorialité de} la psychanalyse dans l'institution psychiatrique

Comment une telle argumentation, consistant finalement, presque explicitement, à se soustraire aux contraintes de justification de la pratique, a-t-elle pu être efficace socialement ? Comment, pour reprendre un terme utilisé par les psychanalystes, une certaine « extraterritorialité » a-t-elle pu être reçue socialement [Lazarovici, 2004] ? Différents éléments spécifiques à la période des années 1960 et du début des années 1970 nous permettent de répondre à cette question. D'une part, le contexte social général - les événements de Mai 68, et, plus spécifique, la réforme de la psychiatrie -, est propice au changement et à l'expérimentation et rend possible une certaine mise en suspens des contraintes d'institutionnalisation et de généralisation de la pratique. Les expériences qui rendent compte de la possibilité de faire entrer des psychanalystes dans les institutions psychiatriques, comme celle de La Borde, celle du XIII ${ }^{\mathrm{e}}$ arrondissement à Paris, ou de Santé mentale et Communauté dans la banlieue lyonnaise, sont expérimentales.

Cette extraterritorialité est rendue possible d'un point de vue administratif. Avant les années 1970, le statut des psychothérapies, à l'intérieur de l'hôpital, n'est pas encore institué. Le psychanalyste peut être présent dans l'équipe sans y avoir une place clairement définie au regard des autres professionnels. Il peut être recruté à l'intérieur de l'hôpital en tant que médecin ou psychologue, voire sociologue... Le statut des médecins dans les hôpitaux favorise également une certaine ambiguïté. Ceux-ci peuvent intervenir à plein temps dans le service public hospitalier tout en conservant une 
clientèle privée. De nombreux chefs de service se présenteront ainsi comme «psychiatre-psychanalyste » sans qu'on sache si ce titre désigne leur double fonction de praticien dans le service public et en clientèle privée ou s'il réfère à l'approche psychanalytique spécifique qu'ils développent en tant que psychiatre. Cette ambiguïté favorise une extraterritorialité « professionnelle » qui est rendue possible par une gestion souple des règles administratives.

Enfin, la prétention à la singularité portée par les psychanalystes et l'autorité [Lézé, 2010], qu'une telle prétention favorise, rencontre un écho auprès d'une profession qui cherche à préserver son identité dans un contexte de critique forte de l'asile. Ce prestige conduit un nombre important d'internes en psychiatrie et de jeunes psychiatres à devenir dans le même temps psychanalystes. De nombreux psychiatres suivent alors une formation psychanalytique. En 1965, dix des trente-trois chefs de service psychiatrique de la région parisienne seraient de formation psychanalytique [Mises, 1965 , p. 212]. À la fin des années 1960, cette importance de la psychanalyse pour la formation des psychiatres est officiellement reconnue à l'intérieur du Certificat d'études spéciales de psychiatrie qui laisse une place prépondérante à la « psychopathologie psychanalytique » [Widlöcher, 2004, p. 12].

\section{L'émergence d'un nouveau référentiel de valorisation du soin : la comparaison}

Assez rapidement cependant, les ingrédients qui rendaient possible le succès de la psychanalyse vont disparaître. La déspécification du soin psychiatrique, d'un point de vue légal et réglementaire, et l'inscription de cette activité dans les transformations de l'organisation des services de santé conduit à l'émergence d'une nouvelle manière de valoriser le soin, non plus à partir de la signification qu'il peut revêtir pour le malade mais à partir de son évaluation socio-économique. Ce basculement s'opère d'abord en raison du contexte historique. Alors que, dans la période de forte croissance économique des années 1950 et 1960, on acceptait que les dépenses de santé connaissent une augmentation exponentielle, l'entrée dans une période de crise économique rend ces dépenses problématiques. C'est ainsi que le souci politique de maîtrise des dépenses 
s'impose peu à peu à partir de la fin des années 1970. Il conduit à l'émergence d'un questionnement économique dans les politiques de santé et à l'introduction d'un nouveau modèle d'évaluation du soin [Benamouzig, 2005].

\section{La déspécification de la pratique psychiatrique et la dissociation du thérapeutique et de l'hébergement}

Au moment même où l'influence de la psychanalyse semble au plus haut, se dessinent des transformations pratiques et institutionnelles profondes dans l'organisation du soin psychiatrique qui ont pour effet de déspécifier cette activité. Une déspécification avait déjà été engagée en pratique à la suite de la mise en place de services ouverts et de la reconnaissance de la maladie mentale comme affection de longue durée, au même titre que d'autres affections, par le décret du 27 juin 1955. En matière d'hospitalisation, les patients pris en charge relèvent de moins en moins souvent du régime spécifique de la loi de 1838 . Les services dits ouverts se sont multipliés au cours des années 1950 et 1960, ce qui permet aux malades d'être soumis à un régime ordinaire d'hospitalisation, au point qu'ils constituent la majorité des hospitalisations au milieu des années 1970. Cette évolution est favorisée par la montée en charge de la Sécurité sociale. La prise en charge des frais d'hospitalisation par l'assurance maladie facilite en effet les demandes de soin par le malade lui-même dans un service psychiatrique. Des évolutions législatives et organisationnelles viennent soutenir et renforcer ces transformations déjà effectives en pratique. La réforme du droit civil en 1968 réduit l'exceptionnalité du régime des patients hospitalisés sans leur consentement. Surtout, la mise en place de la politique de sectorisation ouvre le soin psychiatrique à de nombreux patients pour qui l'hospitalisation n'est que de courte durée ou dont l'ensemble du traitement est assuré en ambulatoire. Cette politique décidée administrativement en 1960 est progressivement organisée. L'activité de soin psychiatrique assurée à l'extérieur de l'hôpital se développe fortement. Il faudra cependant attendre le milieu des années 1980 pour que son financement relève, à l'instar de l'activité intra-hospitalière, de l'assurance-maladie. 
La déspécification de l'activité psychiatrique est également mise en œuvre par un changement de statut des établissements hospitaliers et de leur personnel. La loi du 31 juillet 1968 intègre les hôpitaux psychiatriques au cadre des hôpitaux généraux. Cette réforme rend possible une transformation de l'organisation du travail dans les établissements qui se caractérise par une segmentation plus importante des différentes activités de prise en charge des malades et des professions impliquées. Jusqu'alors, un même statut était partagé par l'ensemble des infirmiers psychiatriques. L'instauration de règles communes aux hôpitaux généraux et aux hôpitaux psychiatriques permet d'institutionnaliser l'arrivée de nouvelles professions à l'intérieur des hôpitaux. Au bas de l'échelle, par exemple, des agents de service hospitalier sont recrutés pour assurer les différentes tâches ménagères jusqu'alors effectués par les malades sous le contrôle et la responsabilité des infirmiers. Le 3 décembre 1971, le décret Boulin instaure un statut pour les psychologues des hôpitaux, qui autonomise et institue leur place. En quelques années, le nombre de psychologues augmente considérablement, aussi bien en intra- qu'en extrahospitalier. D'autres professions paramédicales sont également introduites : ergothérapeutes, kinésithérapeutes, orthophonistes développent des soins particuliers au sein d'une équipe soignante maintenant pluri-professionnelle.

La mise en place de nouvelles pratiques, l'apparition de nouvelles professions, le changement de lieux du soin soulèvent des enjeux de financement qui conduisent les autorités administratives à établir une séparation plus claire entre ce qui relève du soin et ce qui n'en relève pas. Là encore, différentes réformes législatives votées à un rythme rapproché dessinent une nouvelle conception du soin. La loi du 30 juin 1975 distingue le financement des activités sanitaires d'un côté, sociales et médico-sociales de l'autre. La loi du 4 janvier 1978 remplace les établissements de « convalescence, cure, et réadaptation », en « unités de moyen (pour convalescence, cure, réadaptation ou traitement des maladies mentales) ou de long séjour pour les personnes n'ayant plus "leur autonomie de vie" » et établit un financement différent pour le soin et pour l'hébergement. La création du forfait journalier en 1984 généralise cette distinction. 


\section{La maîtrise des dépenses de santé et la mise en comparaison du soin}

À partir de la fin des années 1970, c'est le souci de la maîtrise des dépenses de santé qui devient l'orientation politique principale. La mise en œuvre d'un tel objectif se confronte cependant à deux difficultés : d'une part, les aspirations sociales vis-à-vis de la santé sont fortes ; d'autre part, le corps médical est réticent à introduire des considérations économiques dans les pratiques de soin. La réponse politico-administrative apportée à cette double contrainte est de mettre en avant la possibilité d'améliorer la gestion des dépenses sans affecter la qualité du soin. Cet argument repose d'abord sur l'existence de nouveaux outils de gestion qui ont été mis en place dans l'administration à la fin des années 1960, comme les protocoles de rationalisation des choix budgétaires (RCB).

Le développement de travaux d'économie de la santé a ensuite facilité l'adaptation de ces outils aux questions de santé [Benamouzig, 2005]. Ils sont introduits dans les hôpitaux généraux et les hôpitaux psychiatriques en deux temps. Dans un premier temps, les outils, proposés puis imposés, sont destinés à réorganiser la gestion administrative des établissements. Dans un second temps, ils visent à favoriser une prise en compte du souci gestionnaire au sein même de l'activité médicale. À partir du milieu des années 1970 se développe une critique forte du mode de financement des hôpitaux qui repose depuis plusieurs décennies sur la fixation d'un prix de journée révisable régulièrement par les autorités administratives en fonction des demandes des établissements. Dans un tel système, les établissements ont intérêt à disposer du plus grand nombre de lits et à garder les patients hospitalisés le plus longtemps possible pour bénéficier d'un financement plus important. Afin de lutter contre ces effets inflationnistes, le financement par le budget global est instauré en 1983.

Cette réforme n'est pas seulement technique. Elle vise également à réorganiser les responsabilités de gestion à l'intérieur de l'hôpital. Elle s'accompagne, d'abord à titre expérimental, puis de manière généralisée, de la mise en place d'une comptabilité analytique qui permet d'isoler le coût de différentes composantes de l'activité hospitalière : les prestations administratives, logistiques, médico- 
techniques et cliniques sont distinguées et décomposées. Cet outil comptable, développé depuis longtemps dans le monde industriel, se diffuse à partir des années 1970 dans les services et dans l'administration publique. Il vise d'abord à offrir des critères détaillés de comparaison dans le temps, d'une année sur l'autre, mais aussi entre les différents « centres de décision » existant au niveau d'un établissement. Son instauration s'accompagne très vite de la mise en place de comparaison entre les établissements hospitaliers. Des fiches d'analyse d'écarts par activités normalisées sont développées. Dès 1987, les «Tableaux de synthèse par activités » (TSA), demandés par la direction générale des hôpitaux, permet aux autorités de tutelle de comparer les coûts entre les différents établissements et de diffuser l'information à ces derniers présentés comme des outils de comparaison avec « des perspectives d'utilisation stratégique ». Des « fiches d'analyses d'écart » ont pour but de permettre aux établissements qui le souhaitent de se comparer à un échantillon d'hôpitaux ayant fait l'effort d'une présentation comptable homogène. La comparaison est non seulement possible sur le coût unitaire total d'une activité mais également sur sa composition, ce qui permet la détermination des causes d'un éventuel surcoût.

L'introduction progressive d'une logique gestionnaire, portée par l'administration, est justifiée par une préoccupation de réduction des inégalités de santé, dans une perspective de santé publique. L'article 21 de la loi du 4 février 1995 veille ainsi à « l'égalité des conditions d'accès au soin sur l'ensemble du territoire, et au maintien des établissements de proximité ». La logique territoriale adoptée aboutit au renforcement du processus de rapprochement de la psychiatrie au sein du système de santé. Récemment, l'ordonnance du 4 septembre 2003, en instaurant un schéma régional d'organisation sanitaire (SROS) comme outil unique et central de planification, a supprimé la carte sanitaire spécifique pour la psychiatrie. Les territoires de santé qui remplacent les secteurs sanitaires sont définis pour l'ensemble du dispositif de soin, y compris psychiatrique. Le même souci des inégalités de santé se retrouve dans la version «médicale » de la logique gestionnaire qui s'est constituée peu à peu avec l'inscription des établissements hospitaliers dans une perspective de valorisation de l'activité, à laquelle les médecins sont associés. 


\section{L'information médicale : rendre compte de l'activité}

En 1991, la loi hospitalière du 3 juillet, qui crée la notion de « service public hospitalier» et institue la carte sanitaire, marque le passage à une autre période puisqu'elle ouvre à la diffusion du souci gestionnaire auprès du corps médical. Les établissements sont tenus de rendre compte de leur activité. Pour cela, ils doivent «mettre en œuvre des systèmes d'information qui tiennent compte notamment des pathologies et des modes de prise en charge en vue d'améliorer la connaissance et l'évaluation de l'activité et des coûts et de favoriser l'optimisation de l'offre de soins. »

L'activité doit être évaluée selon une méthode spécifique, élaborée progressivement depuis le début des années 1980 dans le cadre du Programme de médicalisation des systèmes d'information (PMSI). Ce programme a été lancé par la direction des hôpitaux en 1982. Il s'agit de confronter l'activité médicale à la consommation de ressources en élaborant des « catégories de séjours hospitaliers » grâce à la constitution de "Groupes homogènes de malades » (GHM) censés articuler une double homogénéité : médicale et économique. Le GHM articule un classement des malades en fonction de leur pathologie, classement se référant à la classification internationale des maladies et un classement en fonction des frais que leur séjour à l'hôpital implique, en personnel et en matériel. L'association d'un séjour à un groupe homogène de malades est réalisée techniquement en remplissant une fiche uniformisée relatant à la sortie les informations relatives au soin d'un patient pendant son hospitalisation. Cette fiche constitue le recueil standardisé de sortie (RSS) qui est envoyé aux autorités de tutelle. Une fois que celles-ci sont inscrites dans des groupes homogènes, on estime sur un échantillon d'hôpitaux et à partir d'une comptabilité analytique standardisée des coûts moyens totaux pour chaque GHM, appelés points ISA (indice synthétique d'activité). À partir de cet indice, un budget « théorique » de chaque établissement peut être établi et la productivité des hôpitaux est évaluée en rapportant les dépenses réelles constatées au nombre total de points ISA. À partir de 1995 , une étude nationale des coûts est menée chaque année qui sert de base à l'élaboration de l'Objectif national des dépenses d'assurancemaladie (ONDAM), voté par la représentation nationale. 
Pour que le système fonctionne, les autorités administratives ont besoin de l'implication des médecins. Celle-ci est obtenue grâce à « un mélange subtil de contrainte et d'incitation » [Lenay et Moisdon, 2003, p. 133] qui se traduit par exemple par des subventions spécifiques pour le recrutement de médecins responsables des départements d'information médicale, rendus obligatoires en 1996. Confier ces départements à une responsabilité médicale a l'intérêt stratégique d'associer les acteurs médicaux de l'établissement au recueil de l'activité [Ogien, 2000]. C'est en 2004 que se met en place la tarification à l'activité. Il s'agit d'un mode de financement qui fonde l'allocation des ressources aux établissements de santé sur la nature et le volume de l'activité réalisée mesuré par le classement de chaque patient dans un groupe homogène de séjour (GHS). Ce nouveau mode de financement concerne alors les hôpitaux dits de « $\mathrm{MCO}$ » pour médecine-chirurgie-obstétrique. Les hôpitaux psychiatriques, quant à eux, restent encore financés par le budget global.

Plus de vingt ans séparent le lancement du programme et l'instauration d'un financement tenant compte de l'activité de chaque hôpital. Malgré les réticences fortes du corps médical, une nouvelle logique fondée sur une évaluation des soins, par la comparaison économique de leur coût et de leurs résultats, s'est profondément imposée dans l'organisation des politiques de santé [Ogien, 2009].

\section{Les résistances spécifiques rencontrées en psychiatrie}

Le processus de rapprochement des hôpitaux psychiatriques du régime hospitalier commun a conduit à ce que ceux-ci soient directement concernés par le développement de ces nouveaux principes d'évaluation du soin. Cependant, l'introduction des nouvelles règles de gestion et de financement rencontre dans ce secteur des difficultés importantes, au point que sera élaboré un PMSI spécifique à la psychiatrie, dont les modalités d'utilisation pour une tarification à l'activité sont encore en discussion.

Le problème de l'évaluation de l'activité présente des dimensions spécifiques dans le champ psychiatrique, dont les répercussions sont particulièrement importantes dans l'organisation du financement du soin. Ces difficultés sont accentuées par la distinction historique du financement du soin entre les hospitalisations, qui sont traditionnellement facturées, et les activités de soin réalisées dans les 
structures extrahospitalières qui ne sont pas facturées aux patients au motif qu'elles relèvent d'une politique de prévention. $\mathrm{Si}$, pendant les années 1950 et 1960, la Sécurité sociale a accepté de participer au financement d'actions expérimentales relevant à la fois de l'hospitalisation, du soin ambulatoire, de la consultation psychothérapique, elle devient très réticente à partir des années 1970 à financer des actes ou des journées qui ne relèvent pas de la nomenclature générale des actes professionnels. Le financement des consultations psychanalytiques privées est âprement discuté pour être finalement accepté au début des années 1970, mais leur prise en charge au titre de l'activité hospitalière est en revanche rejetée $e^{4}$.

Une décision politique impose finalement la prise en charge de l'activité extrahospitalière par la Sécurité sociale en 1986. La Caisse nationale d'assurance-maladie voudrait cependant pouvoir mesurer les soins ambulatoires qu'elle prend en charge. Pour répondre à cette demande, il est demandé aux établissements psychiatriques de mettre en place une fiche par patient qui doit faciliter la constitution du recueil standardisé de sortie (RSS). Cependant, les caractéristiques de l'exercice de la psychiatrie, qui associe un grand nombre de structures aux fonctionnements divers à une équipe rassemblant différents professionnels aux modes d'intervention variés, rendent la mise en œuvre difficile. Dès 1986, la direction générale de la santé se montre prudente : «[...] du fait de la complexité et de la multiplicité d'une part, des actions menées pour un même patient qui peuvent nécessiter la participation de toute une équipe et, d'autre part, des actions non liées directement au patient, une simple comptabilité des actes ne saurait bien rendre compte de cette activité. C'est ainsi que dans le souci de mesurer le service rendu à la population, il apparaît impératif dans un premier temps de comptabiliser très précisément le nombre de malades suivis dans l'année par l'équipe de secteur et d'étudier globalement les modes de prise en charge des patients » (Lettre circulaire $\mathrm{n}^{\circ} 552$ de la DGS, 7 mai 1986).

4. Une expérience menée à l'hôpital du Vinatier est là encore éclairante. À la suite de la proposition d'un chef de secteur, J. Hochman, de faire passer sa clientèle privée à une prise en charge publique hospitalière en créant une « unité de traitement psychanalytique », une demande est faite à la caisse régionale d'assurance-maladie par la direction de l'hôpital de fixer un tarif de financement de l'activité. Celle-ci est refusée par courrier du 25 novembre 1983 en raison des instructions de la Caisse nationale. (Archives de l'hôpital du Vinatier). 
Peu de temps après, la mise en place des Départements d'information médicale (DIM) conduit la direction des hôpitaux à prévoir un PMSI spécifique à la psychiatrie dont la mise en place prendra près de quinze ans. Ce n'est en effet qu'en 2006 que le relevé d'information médicalisé en psychiatrie (RIMP) est rendu obligatoire dans les établissements. Celui-ci prépare la mise en place de la tarification à l'activité, intitulée « Valorisation de l'activité en psychiatrie », qui a pour objectif de pondérer le financement à l'activité par d'autres types de financement. À cette occasion, les psychiatres défendent la nécessité de laisser une part au financement qui tienne compte notamment des caractéristiques géoéconomiques des populations desservies et des missions d'intérêt général assurées par les établissements de soin en psychiatrie. Il s'agit pour eux de concilier les impératifs gestionnaires qui leur sont imposés avec ce qu'ils conçoivent comme les spécificités de la mission du dispositif de soins psychiatriques.

En 2004, dans un document de travail, plusieurs acteurs du champ de la psychiatrie ${ }^{5}$ défendent le maintien d'un financement partiel par dotation par le fait qu'il introduit une « équité républicaine d'accès aux soins pour les populations et renvoie aux missions de secteur en psychiatrie, missions de soins, de prévention et de réinsertion articulées sur le concept de population à prendre en charge. » Le processus long qui est en passe d'aboutir à un financement de la psychiatrie publique par tarification à l'activité, même partiellement, est à la mesure des résistances particulièrement importantes des acteurs de la psychiatrie à ces évolutions. Il associe, comme dans le champ de la santé, la maîtrise des dépenses et la réduction des inégalités de santé.

Avec la mise en place d'outils de maîtrise des dépenses de santé et de dispositifs de recueil de l'activité au sein même des hôpitaux psychiatriques, les conditions de possibilité de l'évaluation comparative des pratiques de soin sont réunies. Ces évaluations sont justifiées par la recherche d'une plus grande équité dans l'organisation de l'offre de soin. Les résistances rencontrées dans la mise

5. Fédération hospitalière de France, FNAP-Psy, UNAFAM, Conférence des présidents de CME, Association des établissements gérant des secteurs de santé mentale, 2004. [En ligne] : <http://www.ch-le-vinatier.com/cme-psy/VAP \% 20succinte- dec04.pdf> 
en œuvre de ces outils dans l'organisation du soin psychiatrique montrent que ce type de soin ne peut complètement relever des politiques générales de santé, quand bien même une dynamique de déspécification du soin psychiatrique s'est instaurée depuis plusieurs décennies.

\section{Conclusion}

En analysant le rôle historique joué par la psychanalyse dans les institutions psychiatriques, on a montré l'importance que cette vision du monde a eu sur toute une génération de psychiatres, vision prometteuse, émancipatrice de la personne humaine, donnant aux professionnels un moteur pour transformer leur institution. Ces transformations se sont cependant peu traduites par l'introduction pratique de la cure psychanalytique à l'intérieur des établissements hospitaliers. Ce décalage entre l'importance symbolique du discours psychanalytique pour les psychiatres et le faible recours à la cure psychanalytique dans les pratiques institutionnelles n'a pas posé problème tant que les conditions sociales de réception de l'extraterritorialité des psychanalystes étaient réunies. Deux conditions relatives au contexte du champ psychiatrique ont été plus particulièrement mises en exergue : le fait qu'il soit propice aux expérimentations en même temps qu'en attente d'une reformulation de l'autorité des psychiatres.

Cette configuration articulant de manière souple vision anthropologique et efficacité thérapeutique s'est affaiblie devant l'émergence de nouvelles évaluations des pratiques thérapeutiques. Progressivement, les logiques gestionnaires se sont imposées, laissant aujourd'hui de nombreux professionnels de la psychiatrie dans un désarroi certain. Provenant d'autres acteurs de la santé (Sécurité sociale, direction des hôpitaux...), parfois reprises par les professionnels eux-mêmes, ces logiques ont mis au centre un souci d'efficacité cherchant à articuler les questions thérapeutiques et économiques. Leur succès a conduit à une réforme profonde dans l'évaluation de l'activité de soin.

Les réticences particulières rencontrées dans l'institutionnalisation de ce type d'évaluation dans le champ psychiatrique laissent entrevoir la place que jouent encore certainement les valeurs 
promues par la psychanalyse, dont la portée s'étend au-delà d'un indéniable effet de génération [Jacqueline, 2006]. C'est la position défendue par certains analystes qui constatent que la psychanalyse est la théorie la plus accueillante pour « la diversité des cas et des situations cliniques » rencontrées [Thurin, 2008]. Au-delà de l'appréciation de la diffusion de la théorie psychanalytique parmi les psychothérapeutes, cette réflexion invite plutôt à s'interroger sur les conditions sociales contemporaines qui peuvent rendre possible la tenue d'une position « extraterritoriale » au regard des impératifs gestionnaires qui s'imposent aujourd'hui dans les politiques publiques. Au regard de l'exemple succinctement analysé du succès de la psychanalyse dans l'institution psychiatrique, on pourrait penser que cette extraterritorialité ne pourrait s'inventer qu'en se tenant « tout contre » les impératifs médico-gestionnaires actuels.

\section{Références bibliographiques}

Alluard O., Coldefy M., 2005, «Atlas régional de psychiatrie générale. Disparités de l'offre et des territoires d'intervention », Drees, Document de travail, série Études, $n^{\circ} 75$, février.

Benamouzig D., 2005, La Santé au miroir de l'économie, PUF, Paris.

BeRgeret J., 1965, «Les psychothérapies en 1964 », Annexe au rapport moral et administratif de l'hôpital du Vinatier.

Castel R., 1971, «L'institution psychiatrique en question », Revue française de sociologie, vol. 12, $\mathrm{n}^{\circ} 1$, p. 57-92.

Champion F. (dir.), 2008, Psychothérapie et société, Armand Colin, Paris.

Coldefy M. (dir.), 2007, La Prise en charge de la santé mentale, recueil d'études statistiques, La Documentation française, Paris.

Coldefy M., Bousquet F., 2002, «Une typologie des secteurs de psychiatrie générale en 1999 », Drees, Études et Résultats, n 163, mars.

Dexter M. Bullard, 1939, «The Application of Psychoanalytic Psychiatry to the Psychoses », Psychoanalytic Review, vol. XxvI, n 4, p. 526-534.

DiATKine R., 1970, «L'apport de la théorie psychanalytique à la compréhension des maladies mentales et, éventuellement, à l'organisation d'institutions destinées à les traiter », in RACAMIER P. C., Le Psychanalyste sans divan, Payot, Paris.

Ehrenberg A., 2010, La Société du malaise, Odile Jacob, Paris. 
Fromm-Reichman F., 1948, « Notes sur l'évolution des traitements des schizophrènes pour la psychothérapie analytique », in Psychiatry, II, 263-273 ; in Revue Française de psychanalyse, XXII, n 1, janv.-fév. 1958, p. 37-58.

Gauchet M. (en collaboration avec G. Swain), 1980, La Pratique de l'esprit humain : l'institution asilaire et la révolution démocratique, Gallimard, Paris.

HencKes N., 2005, « Réformer la psychiatrie, organiser les pratiques de secteur. La construction de la psychiatrie de secteur dans "l'expérience du treizième arrondissement" », Rapport pour le programme Sciences biomédicales, Santé, Société, MiRe/INSERM/CNRS, CERMES, Paris.

INSERM, 2004, Psychothérapie, trois approches évaluées, février.

JACQUELINE S., 2006, « Les politiques du patient en pratique. Psychanalyse et psychopharmacologie à l'hôpital », Politix, vol. 19, nº 73.

LAZAROVICI R., 2004, « La psychanalyse : extraterritorialité maintenue ou dilution en psychiatrie de secteur ? », Topique, $3, \mathrm{n}^{\circ} 88$.

Lebovici S., 1970, « La psychanalyse et la psychiatrie d'aujourd'hui », in RaCAmier P. C. et alii, Le Psychanalyste sans divan, Payot, Paris.

Lenay, O., Moisdon, J.-C., 2003, « Du système d'information médicalisée à la tarification à l'activité. Trajectoire d'un instrument de gestion du système hospitalier », Revue française de gestion, $\mathrm{n}^{\circ}$ 146, mai, p. 131-141.

LÉzé S., 2010, L'Autorité des psychanalystes, PUF, Paris.

Mannoni M., 1970, Le Psychiatre, son fou, et la psychanalyse, Seuil, Paris.

Mises R., 1965, « Les Psychothérapies. Rapport avec la psychanalyse », Livre Blanc de la psychiatrie française, t. I.

OGIEN A., 2009, « L'hôpital saisi par la quantification. Une analyse de l'usage gestionnaire de la notion de qualité », Sciences de la société, $\mathrm{n}^{\circ} 76$, p. 31-51.

- 2000, « Médecine, santé et gestion. Le travail de l'information médicale », in Cresson G. et Schweyer F. X. (dir.), 2000, Professions et institutions de santé face à l'organisation du travail, Éditions de l'ENSP, Rennes, p. 133-148.

OHAYon A., 2008, « Exercice de la psychothérapie, pouvoir médical et passions françaises dans les années 1950 », in CHAMPION F. (dir.), Psychothérapie et société, Armand Colin, Paris, p. 35-53.

Racamier P. C., avec Diatkine R., Lebovici S. et Paumelle P., 1970, Le Psychanalyste sans divan, Payot, Paris.

Thurin J. M., 2008, «L'évaluation des psychothérapies : la "révolution" en cours », in Champion F. (dir.), Psychothérapie et société, Armand Colin, Paris, p. 236-254.

WIDLÖCHER D., 2004, «Psychanalyse et psychiatrie française. 50 ans d'histoire », Topique, $3, \mathrm{n}^{\circ} 88$, p. 7-16. 